\title{
CRESCIMENTO DE CANA-DE-AÇÚCAR SOB APLICAÇÃO DE BIOFERTILIZANTE DA BOVINOCULTURA E UREIA
}

\author{
Henrique Vieira de Mendonça* \\ Jean Pierre Henry Balbaud Ometto** \\ Wadson Sebastião Duarte da Rocha*** \\ Carlos Eugênio Martins**** \\ Marcelo Henrique Otenio***** \\ Cristiano Amancio Vieira Borges ${ }^{* * * * * *}$
}

RESUMO: Os efluentes gerados na pecuária intensiva quando tratados por reatores anaeróbios possuem relevante potencial para reuso agrícola. Este trabalho teve como objetivo avaliar o crescimento das cultivares de cana-de-açúcar SP 803280 e RB 867515 durante 220 dias. Foram aplicadas doses de 0, 16, 48, 64, 80, $96 \mathrm{~kg} \mathrm{ha}^{-1}$ de nitrogênio, usando como fontes biofertilizantes produzidos por meio da água residuária da bovinocultura e ureia. $\mathrm{O}$ delineamento experimental utilizado foi $\mathrm{o}$ de blocos ao acaso, totalizando 96 parcelas com quatro repetições. As cultivares adubadas com biofertilizante obtiveram crescimento semelhante às adubadas com ureia, onde as doses de 64, 80, $96 \mathrm{~kg} \mathrm{ha}^{-1}$ proporcionaram melhor eficácia no crescimento. Ao fim do experimento obteve-se uma altura média de três metros para as culturas, indicando que a ureia pode ser substituída pelo biofertilizante.

PALAVRAS-CHAVE: Biofertirrigação; Dejetos de bovinos; Nitrogênio.

Doutorando em Ecologia Aplicada ao Manejo e Conservação de Recursos Naturais pela Universidade Federal de Juiz de Fora, Departamento de Biologia/Programa de Pós-graduação em Ecologia (PGECOL), Brasil.

** Pesquisador titular e Coordenador do Centro de Ciências do Sistema Terrestre do Instituto Nacional de Pesquisas Espaciais (CST/INPE); Coordena Convênio de Colaboração e Apoio ao International GeosphereBiosphere Program (IGBP) e Global Land Program (GLP), no Brasil.

${ }^{* * *}$ Doutor em Agronomia pela Escola Superior de Agricultura 'Luiz de Queiroz (ESALQ/USP); Pesquisador na da Empresa Brasileira de Pesquisa Agropecuária (EMBRAPA Gado de Leite), Juiz de Fora (MG), Brasil;

E-mail: wadson.rocha@embrapa.br

**** Doutor em Agronomia pela Universidade Federal de Viçosa. Pesquisador A da Empresa Brasileira de Pesquisa Agropecuária (EMBRAPA Gado de Leite), Juiz de Fora (MG), Brasil.

***** Doutorado em Ciências Biológicas pela Universidade Estadual Paulista (UNESP), Rio Claro (SP); Pesquisador A, na área de gestão ambiental e recursos hídricos e efluentes da Empresa Brasileira de Pesquisa Agropecuária (EMBRAPA Gado de Leite), Juiz de Fora (MG), Brasil.

****** Mestrado em Estatística pela Universidade Estadual de Campinas (UNICAMP), Campinas; Analista A da Empresa Brasileira de Pesquisa Agropecuária (EMBRAPA Gado de Leite), Juiz de Fora (MG), Brasil. 


\section{SUGARCANE GROWTH WITH CATTLE BIOFERTILIZER}

\section{AND UREA}

ABSTRACT: Effluents from intensive cattle breeding and treated with anaerobic reactors are highly relevant for agricultural reuse. Current paper assesses the growth of sugarcane cultivars SP 803280 and RB 867515 for 220 days. Doses 0, 16, 48, 64, 80 and $96 \mathrm{~kg} \mathrm{ha}^{-1} \mathrm{~N}$ were applied derived from biofertilizing sources produced by cattle-breeding waste water and urea. Experimental design comprised randomized blocks with 96 splits and four replications. The cultivars fertilized with biofertilizers caused growth similar to those fertilized with urea. Doses 64,80 and $96 \mathrm{~kg} \mathrm{ha}^{-1}$ had the best efficiency in growth. Since average height of $3 \mathrm{~m}$ was obtained for cultures at the end of the experiment, urea may be replaced by biofertilizers.

KEY WORDS: Nitrogen; Biofertirrigation; Cattle wastes.

\section{INTRODUÇÃO}

A pecuária de leite representa relevante importância para a economia mundial. Em 2013, o Brasil produziu 32,3 bilhões de litros de leite, atingindo o quarto lugar no ranking mundial do produto (MAPA, 2014).

Por outro lado, esta atividade pode causar sérios impactos ambientais, em especial aos recursos hídricos, pelo lançamento de efluentes não tratados. Segundo Matos (2014) o confinamento de 1000 cabeças de bovinos resulta na produção de $4200 \mathrm{t}$ de resíduos sólidos e $75 \mathrm{~m}^{3}$ de resíduos líquidos por dia. Considerando a DBO de $10.000 \mathrm{mg} \mathrm{L}^{-1}$ (VON SPERLING, 2005) e uma geração de $75 \mathrm{~L} \mathrm{dia}^{-1} \mathrm{de}$ efluentes por animal, uma unidade de criação intensiva com 1000 matrizes possui equivalência à uma população de 13.890 pessoas, evidenciando a necessidade do tratamento da água residuária da bovinocultura (ARB), antes de sua disposição final.

Uma das técnicas atuais para disposição destes resíduos é a produção de biofertilizante nitrogenado para aplicação no solo para fins agrícolas.

Segundo Silva et al. (2012) o aproveitamento de águas residuárias na fertirrigação de culturas é uma opção recomendada para a redução da poluição ambiental, além de promover melhoria nos atributos do solo e economia com a adubação convencional. 
Estão contidos nos efluentes da bovinocultura macro e micronutrientes essenciais a culturas agrícolas, e que podem ser utilizados na agricultura de forma controlada (ANDRADE FILHO et al., 2013; ERTHAL et al., 2010).

Visando suplementar informações sobre adubação alternativa, o objetivo desta pesquisa foi avaliar a curva de crescimento de duas cultivares de cana-deaçúcar, SP 803280 e RB 867515, submetidas a doses de nitrogênio fornecidas por biofertilizante e adubação convencional com ureia.

\section{MATERIAL E MÉTODOS}

O experimento foi conduzido na área experimental da Embrapa Gado de Leite, município de Coronel Pacheco (MG), nas coordenadas geográficas: $21^{\circ} 33^{\prime}$ 58" S; $43^{\circ} 15^{\prime} 21^{\prime \prime} \mathrm{O}$, a $445 \mathrm{~m}$ de altitude.

O clima da área é classificado como Cwa, segundo Köppen \& Geiger, com temperatura média de $21,7^{\circ} \mathrm{C}$, máxima de $29,4^{\circ} \mathrm{C}$ e mínima de $18,1^{\circ} \mathrm{C}$, apresentando $1.516 \mathrm{~mm}$ de pluviosidade média anual. O solo da área é classificado como nitossolo vermelho, com textura franco argilo arenosa.

Foram realizadas amostragens de solo em três profundidades (Tabela 1), conforme o manual de métodos de análise de solo (EMBRAPA, 1997).

Tabela 1. Atributos médios do solo em diferentes profundidades

\begin{tabular}{l|l|l|l}
\hline Atributos & P 1 & P 2 & P 3 \\
\hline $\mathrm{pH}\left(\mathrm{H}_{2} \mathrm{O}\right)$ & 5,0 & 5,1 & 5,5 \\
\hline $\mathrm{P}\left(\mathrm{mg} \mathrm{dm}^{-3}\right)$ & 3,6 & 2,8 & 3,0 \\
\hline $\mathrm{K}\left(\mathrm{mg} \mathrm{dm}^{-3}\right)$ & 63 & 43 & 18 \\
\hline $\mathrm{Na}\left(\mathrm{mg} \mathrm{dm}^{-3}\right)$ & 7,0 & 7,0 & 7,0 \\
\hline $\mathrm{Ca}\left(\mathrm{cmol}_{\mathrm{c}} \mathrm{dm}^{-3}\right)$ & 2,3 & 2,3 & 2,4 \\
\hline $\mathrm{Mg}\left(\mathrm{cmol}_{c} \mathrm{dm}^{-3}\right)$ & 1,0 & 1,0 & 0,7 \\
\hline $\mathrm{Al}\left(\mathrm{cmol}_{c} \mathrm{dm}^{-3}\right)$ & 0,0 & 0,0 & 0,0 \\
\hline $\mathrm{H}+\mathrm{Al}\left(\mathrm{cmol}_{c} \mathrm{dm}^{-3}\right)$ & 4,95 & 4,46 & 2,15 \\
\hline $\mathrm{SB}\left(\mathrm{cmol}_{\mathrm{c}} \mathrm{dm}^{-3}\right)$ & 3,49 & 3,44 & 3,18 \\
\hline $\mathrm{CTC}\left(\mathrm{cmol}_{c} \mathrm{dm}^{-3}\right)$ & 8,44 & 7,90 & 5,33 \\
\hline $\mathrm{V}(\%)$ & 60 & 51 & 44 \\
\hline
\end{tabular}


(conclusão)

\begin{tabular}{l|l|l|l}
\hline Atributos & P 1 & P 2 & P 3 \\
\hline ISNa (\%) & 0,87 & 0,88 & 0,96 \\
\hline MO (dag Kg $)$ & 2,75 & 1,97 & 1,35 \\
\hline Argila (\%) & 18 & 25 & 43 \\
\hline Silte (\%) & 18 & 17 & 13 \\
\hline Areia (\%) & 64 & 58 & 44 \\
\hline$\rho\left(\mathrm{t} \mathrm{m}^{-3}\right)$ & 1,28 & 1,32 & 1,30 \\
\hline
\end{tabular}

Nota: P1: 0-20 cm; P2: 20-40 cm; P3: 40-60 cm.

O biofertilizante utilizado na pesquisa foi produzido na própria área experimental, por meio da ARB gerada na limpeza de um free stall (Figura 1).

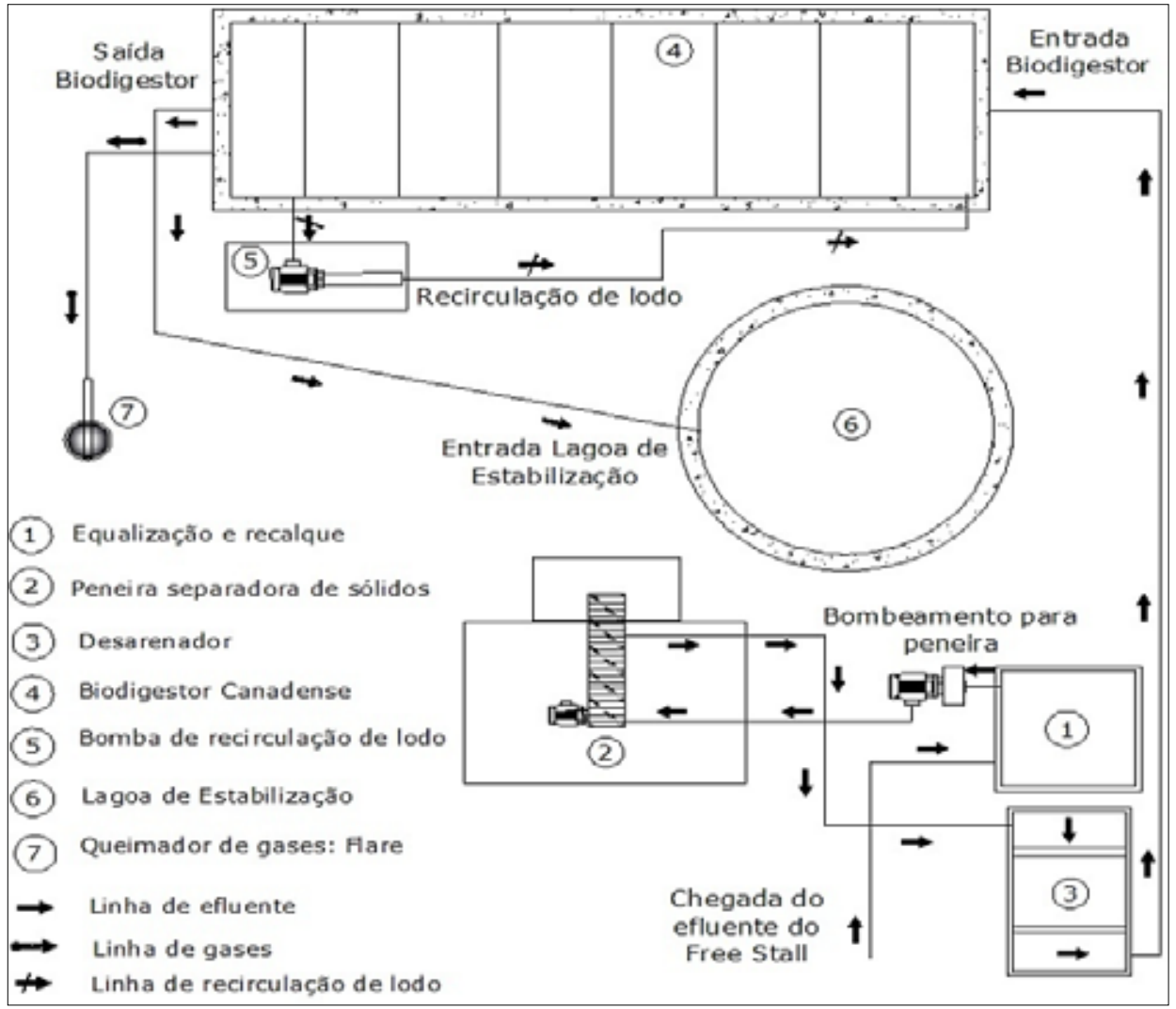

Figura 1. Fluxograma do processo de produção do biofertilizante 
A ARB inicialmente escoa até um tanque de equalização (1), posteriormente é bombeada para uma peneira centrífuga separadora de sólidos (2). Em seguida a ARB escoa para o desarenador (3). Após a desarenação a ARB segue para o biodigestor canadense (4) que opera com um tempo de detenção hidráulica (Tdh) médio de 70 dias. No biodigestor ocorre recirculação do lodo, por meio de bomba helicoidal (5). Os gases gerados no biodigestor são queimados em um sistema tipo flare (7). Após a digestão anaeróbia, a ARB segue para a lagoa de estabilização, com Tdh de 07 dias (6), findando o processo de produção do biofertilizante.

Foram realizadas amostragens quinzenais, por um ano, na lagoa de estabilização (6) sendo as amostras encaminhadas para o laboratório de análises de resíduos da Embrapa Gado de Leite (Tabela 2), executadas em conformidade com APHA et al. (2005).

Tabela 2. Atributos físico-químicos do biofertilizante

\begin{tabular}{lcccc}
\hline Atributos & Média & Máximo & Mínimo & DP \\
\hline pH & 7,0 & 8,0 & 7,0 & $\pm 0,2$ \\
DBO & 637 & 850 & 350 & \pm 171 \\
DQO & 3.396 & 7.316 & 2.188 & \pm 1.773 \\
Norgânico & 22,46 & 36,59 & 3,23 & $\pm 12,5$ \\
Namoniacal & 29,13 & 56,12 & 15,39 & \pm 16 \\
Nitrito & 0,07 & 0,16 & 0,01 & $\pm 0,05$ \\
Nitrato & 42,58 & 59,90 & 24,75 & $\pm 14,3$ \\
Ninorgânico & 71,77 & 96,44 & 44,29 & $\pm 18,8$ \\
Ntotal & 94,23 & 126,7 & 69,95 & \pm 21 \\
Ptotal & 31,64 & 56,88 & 16,55 & $\pm 14,5$ \\
Fosfato & 13,73 & 21,17 & 7,24 & $\pm 5,6$ \\
Ktotal & 0,2 & 0,5 & 0,1 & $\pm 0,2$ \\
Mg & 0,05 & 0,1 & 0,0 & $\pm 0,05$ \\
Na & 0,01 & 0,01 & 0,01 & $\pm 0,0$ \\
Zn & 1,0 & 1,8 & 0,35 & $\pm 0,5$ \\
Ca & 0,05 & 0,1 & 0,0 & $\pm 0,05$ \\
Fe & 19,8 & 36 & 7,0 & $\pm 12,7$ \\
\hline
\end{tabular}

Valores em mg L L ${ }^{-1}$ exceto pH. DP: Desvio Padrão. 
No biofertilizante há maior quantidade de compostos nitrogenados que os demais nutrientes analisados. Portanto, foi calculada a taxa de aplicação (dose), com base na metodologia proposta por Matos (2014), expressa pela Equação 1.

$$
D A_{A R}=\frac{1000 \times\left[N_{a b s}-\left(\operatorname{Tm} 1 \times M O \times \rho_{s} \times p \times 10^{7} \times 0,05 \times \frac{n}{12}\right)\right]}{\left[\left(T m 2 \times \frac{n}{12} \times N_{\text {org }}\right)+\left(N_{\text {amoniacal }}+N_{\text {nitrato }}\right) \times T R\right]}
$$

Em que:

$\mathrm{DA}_{\mathrm{AR}}$ : taxa de aplicação ou dose, $\mathrm{m}^{3} \mathrm{ha}^{-1}$;

$\mathrm{N}_{\mathrm{abs}}$ : absorção de nitrogênio pela cultura, $\mathrm{kg} \mathrm{ha}^{-1}$;

Tm1: taxa anual de mineralização da MO no solo, $\mathrm{kg} \mathrm{kg}^{-1}$ ano $^{-1}$;

MO: conteúdo de matéria orgânica do solo, $\mathrm{kg} \mathrm{kg}^{-1}$;

rs: massa específica do solo, $\mathrm{t} \mathrm{m}^{-3}$;

P: profundidade do solo considerada, $\mathrm{m}$;

$\mathrm{n} / 12$ : fração anual relativa ao período de cultivo, ano;

Tm2: taxa de mineralização do nitrogênio orgânico, $\mathrm{kg} \mathrm{kg}^{-1}$ ano-1;

$\mathrm{N}_{\text {org }}$ : nitrogênio orgânico, $\mathrm{mg} \mathrm{L}^{-1}$;

$\mathrm{N}_{\text {amoniacal }}$ : nitrogênio amoniacal, $\mathrm{mg} \mathrm{L}^{-1}$;

$\mathrm{N}_{\text {nitrato }}$ : nitrogênio nítrico, $\mathrm{mg} \mathrm{L}^{-1} ; \mathrm{e}$

TR: taxa de recuperação do nitrogênio mineral pela cultura, $\mathrm{kg} \mathrm{kg}^{-1} \mathrm{ano}^{-1}$.

As taxas anuais de mineralização da matéria orgânica, nitrogênio orgânico e nitrogênio mineral recuperados pela cultura, utilizados na Equação (1) foram 0,01, 0,5 e $0,7 \mathrm{~kg} \mathrm{~kg}^{-1}$ ano $^{-1}$, respectivamente, valores determinados por Matos (2014), para aplicação de dejetos de bovinos no solo. A taxa de aplicação ideal para a cultura da cana-de-açúcar, calculada pela Equação (1), foi de $80 \mathrm{~kg} \mathrm{ha}^{-1}$ de $\mathrm{N}$.

O biofertilizante foi aplicado na superfície do solo, por meio de mangueira adaptada ao reservatório, sendo controlada a lâmina aplicada por sucessivas medições de vazão. 
Devido ao baixo teor de potássio no biofertilizante, em todas as parcelas foi adicionado $120 \mathrm{~kg} \mathrm{ha}^{-1}$ de $\mathrm{K}_{2} \mathrm{O}$, sendo o cloreto de potássio usado como fonte, fracionados em duas aplicações, uma aos 60 e outra aos 90 dias após o plantio (aplicado no mesmo dia que o nitrogênio). Durante o plantio foram aplicados no sulco $100 \mathrm{~kg} \mathrm{ha}^{-1}$ de $\mathrm{P}_{2} \mathrm{O}_{5}$, sendo utilizado o superfosfato simples, complementando o déficit deste elemento no biofertilizante.

A área total do experimento possuiu $1.728 \mathrm{~m}^{2}$, subdividida em 04 blocos de $24 \times 18$ sendo as parcelas de $18 \mathrm{~m}^{2}(03 \times 06 \mathrm{~m})$. O delineamento experimental utilizado foi de blocos ao acaso, em um esquema fatorial de parcela subdividida, totalizando 24 tratamentos ( 02 cultivares de cana, 02 fontes de nitrogênio e 06 doses), com 04 repetições, sendo 96 o número total de parcelas/unidades experimentais. Foram utilizadas as cultivares de cana-de-açúcar SP 803280 e RB 867515, o biofertilizante e ureia como fornecedores de nitrogênio nas doses $0,16,48,64,80,96 \mathrm{~kg} \mathrm{ha}^{-1}$ (Tabela 3).

Tabela 3. Valores de nitrogênio aplicados e fontes de adubação utilizadas

\begin{tabular}{cccccc}
\hline \multicolumn{2}{c}{ Biofertilizante } & \multicolumn{3}{c}{ Ureia - CO $\left(\mathrm{NH}_{2}\right)_{2}$} \\
\hline Lâmina & $\mathrm{kg} \mathrm{ha}^{-1} \mathrm{de} \mathrm{N}$ & Lâmina $(\mathrm{mm})$ & Parcela & $\mathrm{kg} \mathrm{ha}^{-1} \mathrm{de} \mathrm{N}$ & $\mathrm{kg} \mathrm{ha}^{-1}$ de Ureia \\
\hline L5 & 96 & 65 & L5' & 96 & 213 \\
L4 & 80 & 54 & L' & 80 & 178 \\
L3 & 64 & 43 & L3' & 64 & 142 \\
L2 & 48 & 33 & L2' & 48 & 107 \\
L1 & 16 & 22 & L1' & 16 & 35 \\
L0 & 0 & 0 & L0' & 0 & 0 \\
\hline
\end{tabular}

A irrigação com água de manancial foi utilizada quando necessário, de acordo com a evapotranspiração da cultura (ETc) sendo a evapotranspiração de referência (ETo) calculada pelo método de Penman (1948), usando os coeficientes de cultivo (Kc) para as culturas determinados por Bernardo et al. (2008). Até os 220 dias de condução do experimento foram contabilizados $1000 \mathrm{~mm}$ de precipitação, sendo aplicada uma lâmina complementar de $350 \mathrm{~mm}$, utilizando sistema de irrigação por aspersão. 
Durante 220 dias foram medidas as alturas das cultivares, de $10 \mathrm{em} 10$ dias, obtendo-se 1.920 dados médios de altura das cultivares.

Para verificar diferenças entre os tratamentos, procedeu-se às análises de variância (ANOVA), dentro de cada tempo. Para tal foi utilizado o software SAS, versão 9.2. Os gráficos foram elaborados pelo software R, versão 3.1.3. Em todos os testes, diferenças entre médias foram consideradas significativas quando a probabilidade (P) for $\leq 0,05$.

\section{RESULTADOS E DISCUSSÃO}

Os resultados do aumento na altura das cultivares em função do tempo são apresentados na Figura 2 (A); na Figura 2 (B) os perfis de crescimento em relação à dose de $\mathrm{N}$ aplicada; e na Figura 2 (C) os perfis médios de crescimento em relação ao tipo de fonte (biofertilizante ou ureia) correlacionados com o crescimento das cultivares.

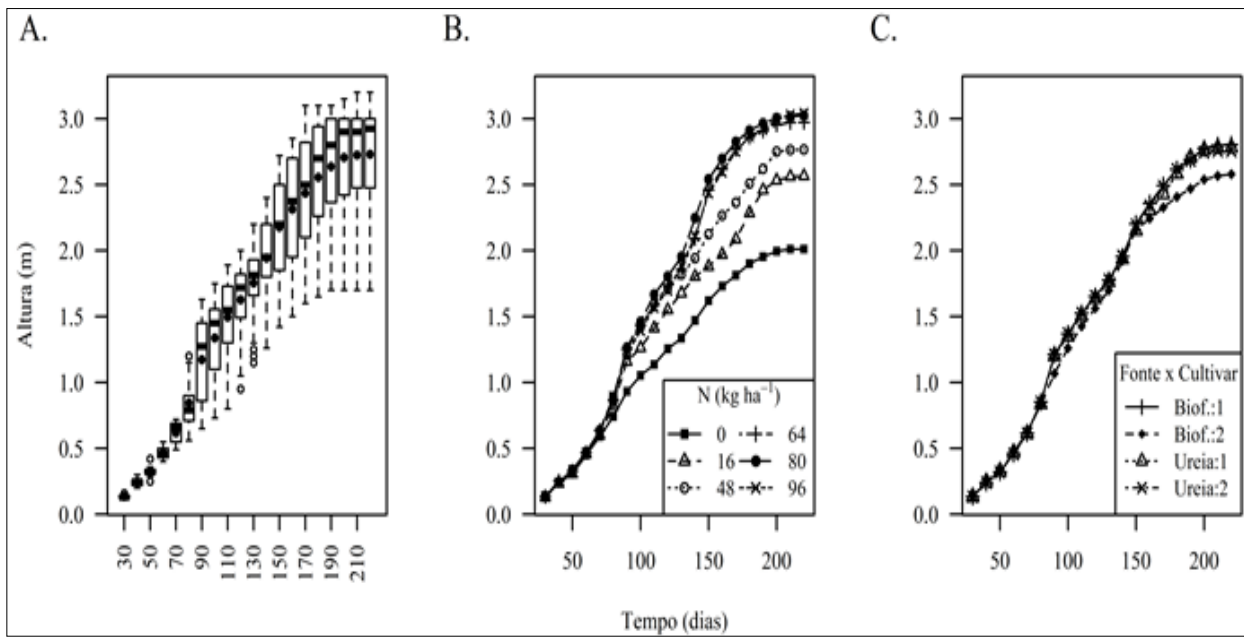

Figura 2. (A) Boxplots para o ganho de altura no tempo. (B) Perfis médios de crescimento em relação às doses de $\mathrm{N}$. (C) Perfis médios de crescimento em relação às combinações de fonte x cultivar, sendo Biof. $=$ Biofertilizante, $1=$ cultivar RB 867515, 2 = cultivar SP 803280.

Observando o crescimento da cana em relação ao tempo, ignorando 
os demais fatores, foi possível identificar três períodos distintos. Até o $80^{\circ}$ dia experimental não houve diferenças $(p>0,05)$ no crescimento das cultivares (Figura $2 \mathrm{~A}$ ), havendo crescimento exponencial, em função do tempo, onde as cultivares atingiram 0,85 m em média. Durante este período as cultivares ainda utilizavam reservas dos colmos plantados. De 90 a 140 dias, houve crescimento linear, também observado por Farias et al. (2008), com variância constante, onde as plantas cresceram em média de 1 a $2 \mathrm{~m}$. De 150 dias a 220 dias, etapa de estabilização no ganho de altura, houve crescimento não linear quadrático, com maior variância, onde as cultivares atingiram em média $2,75 \mathrm{~m}$.

Oliveira et al. (2010) cultivaram a variedade RB 867515, aplicando $30 \mathrm{~kg}$ $\mathrm{ha}^{-1}$ de $\mathrm{N}, 120 \mathrm{~kg} \mathrm{ha}^{-1}$ de $\mathrm{P}_{2} \mathrm{O}_{5}$ e $70 \mathrm{~kg} \mathrm{ha}^{-1}$ de $\mathrm{K}_{2} \mathrm{O}$, respectivamente, na forma de sulfato de amônio, cloreto de potássio e superfosfato triplo, realizando a adubação de cobertura no $90^{\circ}$ dia experimental aplicando $50 \mathrm{~kg} \mathrm{ha}^{-1}$ de $\mathrm{N}$ e $50 \mathrm{~kg} \mathrm{ha}^{-1}$ de $\mathrm{K}_{2} \mathrm{O}$, obtiveram altura da cultivar de 2,75 metros aos 220 dias experimentais, valor semelhante ao deste trabalho. Notadamente a soma da adubação nos sulcos e de cobertura utilizada pelos autores equivalem a $80 \mathrm{~kg} \mathrm{ha}^{-1}$ de $\mathrm{N}$, demonstrando que esta dose de referência nitrogenada, independente do uso do biofertilizante, ureia ou sulfato de amônio proporcionam crescimentos equivalentes para a cultura RB 867515. A dose de $80 \mathrm{~kg} \mathrm{ha}^{-1}$ de $\mathrm{N}$ esteve sempre associada ao maior crescimento das cultivares, confirmando sua frequente consideração como um nível de referência ideal calculada para fontes nitrogenadas.

As doses de 16 e $48 \mathrm{~kg} \mathrm{ha}^{-1}$ de $\mathrm{N}$ proporcionaram menores crescimentos, entre 2,5 e $2,7 \mathrm{~m}$ respectivamente, diferindo-se $(p<0,05)$ em relação às doses 64 , $80,96 \mathrm{~kg} \mathrm{ha}^{-1}$ de N, passados 160 dias (Figura $2 \mathrm{~B}$ ). As parcelas testemunhas (0 kg $\mathrm{ha}^{-1} \mathrm{de} \mathrm{N}$ ) atingiram alturas abaixo de $2 \mathrm{~m}$, em média $1,75 \mathrm{~m}$, indicando a existência da influência das doses de biofertilizante nitrogenado e ureia no crescimento das cultivares (Figura 2 B).

Verificou-se ainda que não há efeito da dose de nitrogênio até 100 dias da brotação, após decorrido este tempo é possível verificar que o crescimento é menor na testemunha em relação às demais doses. A partir de 160 dias é possível verificar diferenças entre as maiores doses $\left(64,80,96 \mathrm{~kg} \mathrm{ha}^{-1}\right)$ e as menores $\left(0,16,48 \mathrm{~kg} \mathrm{ha}^{-1}\right)$. Analisando a relação fonte nitrogenada x cultivar (Figura 2 C), a cultivar SP 803280, obteve queda na altura iniciada no $160^{\circ}$ dia em relação à $\mathrm{RB} 867515$, sendo evidente 
para o biofertilizante.

Batista et al. (2013) conduziram um experimento por 230 dias, visando avaliar a modelagem do crescimento de cultivares de cana-de-açúcar no período de formação da cultura. Os autores utilizaram as variedades RB 92579, RB 867515, RB 928064 e RB 855453, com uso de adubação no plantio, por meio de $45 \mathrm{~kg} \mathrm{ha}^{-1}$ de $\mathrm{N}, 120 \mathrm{~kg} \mathrm{ha}^{-1}$ de $\mathrm{P}_{2} \mathrm{O}_{5}$ e $45 \mathrm{~kg} \mathrm{ha}^{-1}$ de $\mathrm{K}_{2} \mathrm{O}$, aplicados nos sulcos e realizando adubação de cobertura aos 60 dias após o plantio com $45 \mathrm{~kg} \mathrm{ha}^{-1}$ de $\mathrm{N}$ e $45 \mathrm{~kg} \mathrm{ha}^{-1}$ de $\mathrm{K}_{2} \mathrm{O}$ nas linhas das plantas. Os maiores valores de crescimento encontrados pelos autores foram das cultivares RB 867515 e RB 855453. Os autores ainda relatam que a cultivar RB 867515 é a mais difundida no Nordeste do Brasil, cujos diversos estudos têm demonstrado sua superioridade em relação a outras cultivares (ALMEIDA et al., 2008; SILVA et al., 2011; OLIVEIRA et al., 2010).

Oliveira et al. (2011) cultivaram as variedades RB 855453 e SP 801816 por 300 dias e obtiveram uma média geral na altura das cultivares de $3,08 \mathrm{~m}$, aplicando diferentes doses de $\mathrm{N} \mathrm{e}_{2} \mathrm{O}$. Os autores relataram que o aumento no crescimento dos colmos com a aplicação de $\mathrm{N}$ é justificada pelo importante efeito do nitrogênio na taxa de perfilhamento das culturas, pelo fato deste elemento intensificar o perfilhamento e o desenvolvimento da cana-de-açúcar, obtendo-se folhas mais longas e largas de coloração verde intensa e menos áspera.

O crescimento das cultivares em função das doses é apresentado na Figura 3, pelos boxplots do aumento de altura em relação às doses de $\mathrm{N}$, para cada tipo de fonte de adubação, em seis instantes de tempo. 


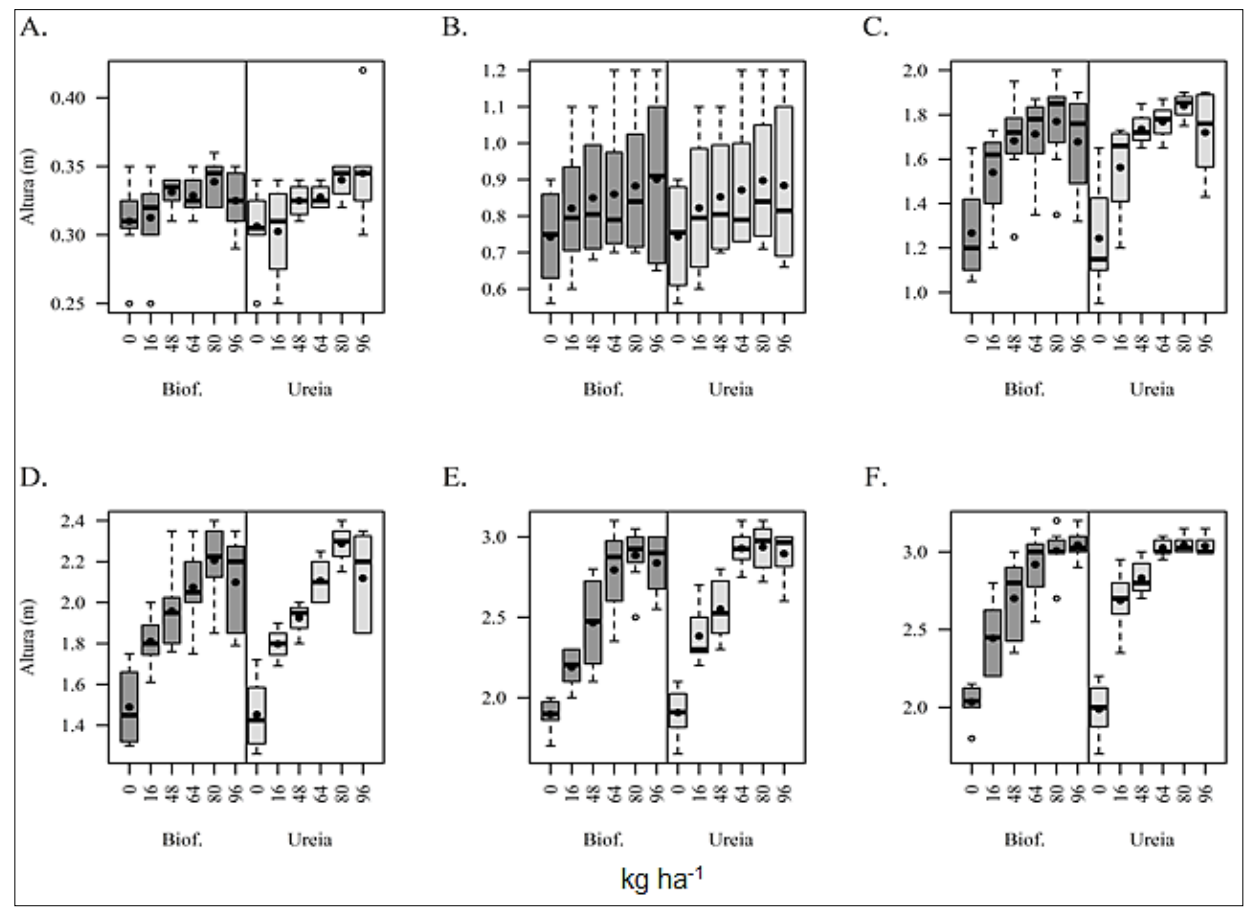

Figura 3. Boxplots para o ganho de altura em relação à dose de $\mathrm{N}$, em cada fonte de adubação, após (A) 50 dias; (B) 80 dias; (C) 120 dias; (D) 140 dias; (E) 180 dias; (F) 220 dias.

Por meio da Figura 4 (A, B e C), foi verificado efeito linear em função das doses, até 80 dias de crescimento, e efeito não linear após 120 dias. A partir do $120^{\circ}$ dia experimental, a dose de referência de $\left(80 \mathrm{~kg} \mathrm{ha}^{-1} \mathrm{de} \mathrm{N}\right)$ foi melhor em relação às demais, verificada até o $180^{\circ}$ dia experimental (Figura $3 \mathrm{E}$ ).

De acordo com a Figura 3 (D, E e F), foi observada equivalência entre as doses $64,80,96 \mathrm{~kg} \mathrm{ha}^{-1} \mathrm{de}$ N. No $220^{\circ}$ dia experimental, os efeitos das doses 64, 80 e $96 \mathrm{~kg} \mathrm{ha}^{-1}$ tornaram-se equivalentes, induzindo as três a uma altura média de $3 \mathrm{~m}$.

Santos (2009) aplicou $66 \mathrm{~kg} \mathrm{ha}^{-1}$ de N, cultivando a variedade RB 75126, e obteve uma altura de $0,47 \mathrm{~m}$ após 04 meses, 1,38 m após 06 meses, 1,85 após 08 meses, usando ureia e cloreto de potássio como adubação de cobertura após 90 dias do plantio. Os menores valores na altura da planta foram atribuídos ao potencial de crescimento intrínseco a cultivar utilizada.

Silva et al. (2014), aplicando $30 \mathrm{~kg} \mathrm{ha}^{-1}$ de nitrogênio (ureia), $120 \mathrm{~kg} \mathrm{ha}^{-1}$ de $\mathrm{P}_{2} \mathrm{O}_{5}$ (superfosfato simples) e $80 \mathrm{~kg} \mathrm{ha}^{-1} \mathrm{de}_{2} \mathrm{O}$ (cloreto de potássio), cultivando 
Saccharum officinarum $\mathrm{L}$., obtiveram alturas de $0,52 \mathrm{~m}$ a 120 dias, $0,80 \mathrm{~m}$ a 140 dias, $1,3 \mathrm{~m}$ a 180 dias e $1,5 \mathrm{~m}$ a 220 dias. Os menores valores na altura da cultivar foram atribuídos à resposta às menores doses de nitrogênio e $\mathrm{K}_{2} \mathrm{O}$ aplicadas. Os autores aplicaram o nitrogênio via água de irrigação parcelado em dez vezes ao longo da condução do experimento, o que pode ter afetado o crescimento por déficit do nutriente em alguma fase do crescimento vegetativo da cultura.

De modo geral as doses de $\mathrm{N}$ equivalentes a 64, 80 e $96 \mathrm{~kg} \mathrm{ha}^{-1}$ apresentaram mesmo efeito de crescimento, com destaque para a dose ideal calculada de 80 $\mathrm{kg} \mathrm{ha}^{-1}$. O uso do biofertilizante nitrogenado nas doses acima de $64 \mathrm{~kg} \mathrm{ha}^{-1} \mathrm{de} \mathrm{N}$ surtiram o mesmo efeito no crescimento das cultivares, quando a ureia foi utilizada, indicando que o biofertilizante pode ser utilizado em substituição à ureia, sem afetar o crescimento das plantas.

\section{CONCLUSÃO}

O biofertilizante (ARB anaeróbiamente digerida) utilizado possui concentração de compostos nitrogenados suficientes para sua utilização no cultivo da cana-de-açúcar.

Não houve diferença no crescimento entre as cultivares em relação ao tempo ou as fontes equivalentes a 64, 80 e $96 \mathrm{~kg} \mathrm{ha}^{-1}$ de adubação nitrogenada utilizada.

As doses 64, 80 e $96 \mathrm{~kg} \mathrm{ha}^{-1}$ de nitrogênio proporcionaram o mesmo efeito no crescimento das cultivares, independente de ser utilizado biofertilizante ou ureia.

$\mathrm{O}$ biofertilizante pode ser utilizado em substituição à ureia para adubação nitrogenada das cultivares RB 867515 e SP 803280.

\section{AGRADECIMENTO}

Os autores agradecem à EMBRAPA Gado de Leite e às agências financiadoras CNPq, Fapemig e CAPES, pelo apoio logístico e financeiro. 


\section{REFERÊNCIAS}

ALMEIDA, A. C. S.; SOUZA, J. L.; TEODORO, I.; BARBOSA, G. V. S.; MOURA FILHO, G.; FERREIRA JÚNIOR, R. A. Desenvolvimento vegetativo e produção de variedades de cana-de-açúcar em relação à disponibilidade hídrica e unidades térmicas. Ciência e Agrotecnologia, Lavras, v. 32, n. 5, p. 1441-1448, 2008.

ANDRADE FILHO, J.; SOUSA NETO, O. N.; DIAS, N. S.; NASCIMENTO, I. B.; MEDEIROS, J. F.; COSME, C. R. Atributos químicos de solo fertirrigado com água residuária no semiárido brasileiro. Irriga, Botucatu, v. 18, n. 4, p. 661-674, 2013.

APHA - American Public Health Association; American Waterworks Association - AWWA; Water Environment Federation - WEF. Standard Methods for the Examination of Water and Waste Water. 21. ed. Washington: APHA; AWWA; WEF, 2005, 1.220 p.

BATISTA, E. L. S.; ZOLNIER, S.; RIBEIRO, A.; LYRA, G. B.; SILVA, T. G. F.; BOEHRINGER, D. Modelagem do crescimento de cultivares de cana-de-açúcar no período de formação da cultura. Revista Brasileira de Engenharia Agrícola e Ambiental, Campina Grande, v. 17, n. 10, p. 1080-1087, 2013.

BERNARDO, S.; SOARES, A. A.; MANTOVANI, E. C. Manual de irrigação. 8. ed. Viçosa: UFV, 2008. 625 p.

DISTRITO FEDERAL. Ministério da Agricultura, Pecuária e Abastecimento - MAPA. Plano mais pecuária, assessoria de gestão estratégica. Brasília: MAPA/ACS, 2014, $32 \mathrm{p}$.

EMBRAPA - Empresa Brasileira de Pesquisa Agropecuária. Manual de métodos de análise de solo. Rio de Janeiro, 1997. 212 p.

ERTHAL, V. J. T.; FERREIRA, P. A.; MATOS, A. T.; PEREIRA, O. G. Alterações físicas e químicas de um argissolo pela aplicação de água residuária de bovinocultura. Revista Brasileira de Engenharia Agrícola e Ambiental, Campina Grande, v. 14, n. 5 , p. $467-477,2010$. 
FARIAS, C. H. A.; FERNANDES, P. D.; AZEVEDO, H. M.; NETO, J. D. Índice de crescimento da cana-de-açúcar de sequeiro no Estado da Paraíba. Revista Brasileira de Engenharia Agrícola e Ambiental, Campina Grande, v. 12, n. 4, p. 356-362, 2008.

MATOS, A. T. Tratamento e aproveitamento agrícola de resíduos sólidos. Viçosa: UFV, 2014, 240 p.

OLIVEIRA, E. C. A.; OLIVEIRA, R. I.; ANDRADE, B. M. T.; FREIRE, F. J.; LIRA JÚNIOR, M. A.; MACHADO, P. R. Crescimento e acúmulo de matéria seca em variedades de cana-de-açúcar cultivadas sob irrigação plena. Revista Brasileira de Engenharia Agrícola e Ambiental, Campina Grande, v. 14, n. 9, p. 951-960, 2010.

OLIVEIRA, F. M.; ASPIAZÚ, I.; KONDO, M. K.; BORGES, I. D.; PEGORARO, R. F.; VIANNA, E. J. Crescimento e produção de variedades de cana-de-açúcar influenciadas por diferentes adubações e estresse hídrico. Revista Trópica - Ciências Agrárias e Biológicas, Chapadinha, v. 5, n. 1, p. 56-67, 2011.

PENMAM, M. L. Evaporation: an introductory survey. Neth. Jour. of Agric. Science, v. 4, p. 9-29, 1948.

SANTOS, V. R.; FILHO, G. M.; ALBUQUERQUE, A. W.; COSTA, J. O. V.; SANTOS, C. G.; SANTOS, A. C. I. Crescimento e produtividade agrícola de cana-de-açúcar em diferentes fontes de fósforo. Revista Brasileira de Engenharia Agrícola e Ambiental, Campina Grande, v. 13, n. 4, p. 389-396, 2009.

SILVA, F. S.; CUNHA, F. N.; OLIVEIRA, R. C.; FREITAS MOURA, L. M.; MOURA, L. C.; TEIXEIRA, M. B. I. Crescimento da cana-de-açúcar sob aplicação de nitrogênio via gotejamento subsuperficial. Revista Brasileira de Agricultura Irrigada, Fortaleza, v. 8 , n. 1, p. 1-11, 2014.

SILVA, J. B. G.; MARTINEZ, M. A.; PIRES, C. P.; ANDRADE, I. P. S.; SILVA, G. T. Avaliação da condutividade elétrica e $\mathrm{pH}$ da solução do solo em uma área fertirrigada com água residuária de bovinocultura de leite. Revista Irriga, Botucatu, edição especial, p. $250-263,2012$. 
SILVA, T. G. F.; MOURA, M. S. B.; ZOLNIER, S.; SOARES, J. M.; VIEIRA, V. J. S.; GOMES JÚNIOR, W. F. Demanda hídrica e eficiência do uso de água da cana-de-açúcar irrigada no semiárido brasileiro. Revista Brasileira de Engenharia Agrícola e Ambiental, Campina Grande, v. 15, n. 12, p. 1257-1265, 2011.

VON SPERLING, M. Princípios de tratamento biológico de águas residuárias: introdução à qualidade das águas e ao tratamento de esgotos. 3. ed. Belo Horizonte: DESA/UFMG, 2005, 425p.

Recebido em: 12 de setembro de 2015 Revisado em: 22 de dezembro de 2015 Aceito em: 21 de janeiro de 2016 